\title{
A Comparison of Data Traffic in Standardized Personal Health Monitoring Solutions
}

\author{
Andreas Schuler, Barbara Franz, and Oliver Krauss
}

\begin{abstract}
The publication of the HL7-FHIR standard offers new possibilities for integrated applications in healthcare. Although trial implementations have only recently started, the application of FHIR in context of a Personal Health Monitoring solution is worth investigating. Most of the existing telemonitoring solutions in healthcare rely on guidelines defined by the Continua Health Alliance (CHA). This paper compares the requirements of CHA and HL7-FHIR with respect to data traffic between client devices and server side applications. Therefore an existing CHAcompliant telemonitoring solution is extended towards supporting HL7-FHIR. Both approaches were simultaneously evaluated in a live system with 68 participants. The results of the evaluation show that the FHIR approach offers the possibility of reducing data traffic in comparison to the CHA solution.
\end{abstract}

Keywords - fast healthcare interoperability resources, integrating the healthcare enterprise, continua health alliance, telemonitoring, electronic health record, SOAP, representational state transfer, m-Health, e-Health

\section{INTRODUCTION}

$\mathbf{P}$ ERSONAL Health Monitoring in connection with the quantified-self movement has recently drawn a lot of attention towards a healthier lifestyle. It is nowadays common for people to use smart watches or one of the many m-Health apps for smartphones to either improve their personal fitness or keep track of their medical condition by measuring vital signs such as blood pressure, weight or heart rate [1]. Considering that cardio vascular diseases in connection with high blood pressure are estimated to cause 7.5 million deaths every year [2], Personal Health Monitoring can not only help to decrease the mortality rate, but it also offers the opportunity to reduce costs in healthcare [1], [3]. Although there is a multitude of different solutions available, there is still room for improvement when it comes to use personal health monitoring as a supplement to healthcare [1].

Nevertheless, most of the existing systems are not applicable for the use in a health environment. The Continua Health Alliance (CHA) offers guidelines for the development of a personal health monitoring solution that satisfies the requirements of current healthcare systems and defines an architecture that is vendor neutral [4]. Relying on standards such as Bluetooth,

Part of this research was conducted in the course of elgaMon, a joint project at the University of Applied Sciences Upper Austria. Its goal was to test the applicability of a personal health monitoring solution in different use cases, in order to estimate its impact on the healthcare system in Austria and to further apply the acquired knowledge to develop a business case.

B. Franz is with the Department of Software Engineering, Upper Austria University of Applied Sciences, Austria, 4232, Hagenberg (e-mail barbara.franz@fh-hagenberg.at).

A. Schuler and O. Krauss are with the Research Department, Uppe Austria University of Applied Sciences, Austria, 4232, Hagenberg (e-mail andreas.schuler@fh-hagenberg.at; oliver.krauss@fh-hagenberg.at).
Health Level Seven (HL7), the WS-* specifications, as well as the profiles defined by Integrating the Healthcare Enterprise (IHE), CHA ensures end-to-end interoperability. The steps involved towards creating a CHA-compliant monitoring solution are outlined in [5], [6]. Although the described solution works well, several problems concerning the compliance to the CHA guidelines could be identified.

As depicted in figure 1 the Aggregation Manager (AM), typically a mobile device e.g. a smartphone, receives a measurement from a personal device, e.g. a blood pressure meter. The measured data is transformed into a HL7v2 message and embedded as payload in a web service request using the Simple Object Access Protocol (SOAP) [4], [7]. Since most mobile devices do not offer an API for developers to interact with SOAP-based web services, SOAP messages have to be created and parsed either manually or using third party libraries, like kSoap for Android [8]. Compared to a lightweight Representational State Transer (REST)-based approach the implementation of a SOAP-based web service client results in increased mobile data traffic, due to the overhead needed to consume a SOAP-based web service from a mobile device [3] and can lead to poor battery life [9].

Current research focuses on the question whether to use SOAP or REST in an environment with limited resources: [9] proposes an architecture for mobile web services based on REST and compare it to the SOAP approach concerning battery-life, data consumption as well as possible limitations. [3] illustrates an architecture for mobile web services called mobWS, that is focused on optimizing HTTP payload for web service calls. [10] shows a RESTful infrastructure for health services to resemble an existing document oriented approach

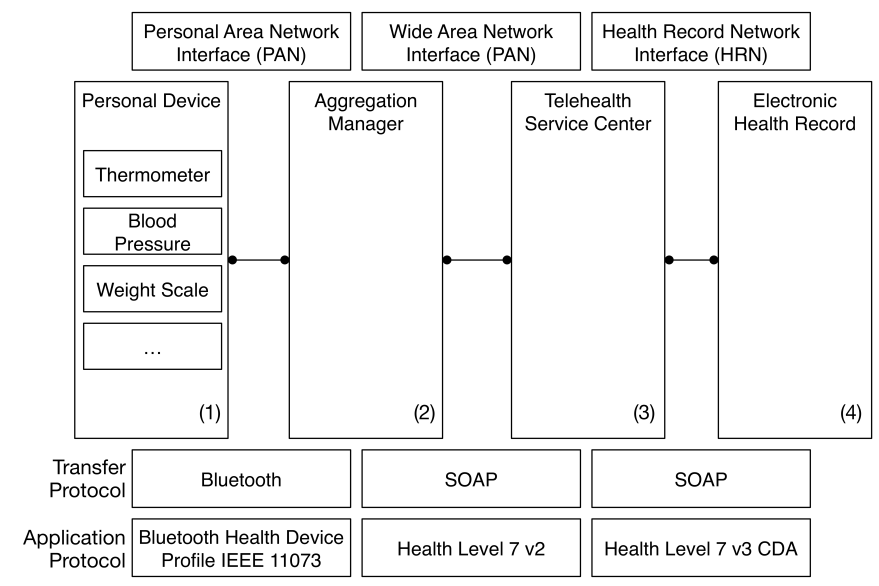

Fig. 1. Components of a CHA-compliant personal health monitoring system as outlined in [3]. 
introducing a lightweight and flexible alternative to the existing SOAP-documents. The authors of [11] compare SOAP and REST regarding performance, efficiency an scalability in order to decide which of the two paradigms is the better choice in course of a Service Oriented Architecture (SOA). [12] concludes from an evaluation comparing the access of a SOAP and a RESTful web service from a smart mobile device, that the latter should be recommended due to its better performance as well as its lower overhead in data. Finally [13] recommends the use of RESTful web services in resource constrained mobile devices, as the evaluation shows that REST outperforms SOAP-based web services in terms of throughput and response time.

Although these approaches seem promising, they neither apply any health standard, nor address use in context of an integrated application in healthcare thus lacking interoperability, demanded by $\mathrm{CHA}$ and affiliated standardization organizations [14]. The new HL7 standard Fast Healthcare Interoperability Resources (FHIR) [15] promises a more flexible approach towards a system for integrated mobile healthcare, compared to its predecessors HL7v2 and HL7v3 [16]

This paper describes an adaption of the interoperable CHAcompliant mobile telemonitoring solution introduced by the authors of this paper proceeding the research from [5], [6] using FHIR, to offer a resource efficient handling of web service connections while still preserving the stipulated interoperability through the use of standards connected to HL7 [17].

\section{METHODS}

The adaption of the CHA compliant approach includes extending the WAN-Interface located between the Aggregation Manager (AM) and the Telehealth Service Center (THCS) (see figure 1) to send measured data according to the HL7-FHIR specification [15]. This is achieved through a set of welldefined data structures, so called FHIR-Resources. These resources enable the development of interoperable web services

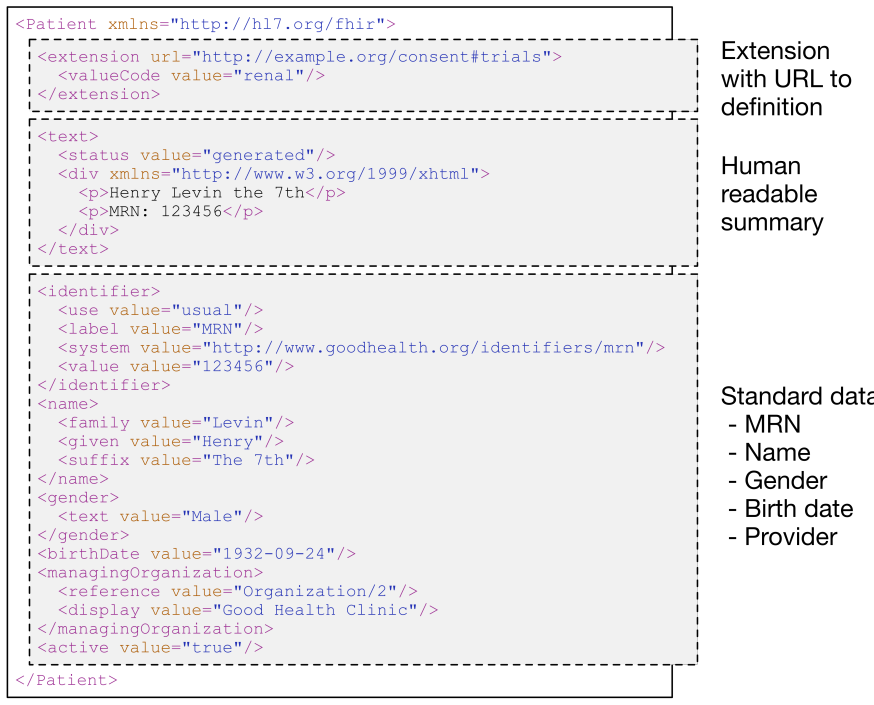

Fig. 2. Fundamental parts of a FHIR-Resource by means of a patient example taken from [15]
MSH|^ \\&||||| $20111215114509.682+0100 \mid$ |ORU^R01^ORU_R01 | 88c3| P | 2.6.1 | | | NE |ALI | | | | PIDIII005.13226488954651^^घ1.2.40.0.10.1.6.1.0.1.100.1.18ISO|I Testpatient^Hage nberg|| $19310320000000+0100 \mid$ M| |

OBR

OBX | | MM | 150021^MDC_PRESS_BLD_NONINV_SYS^MDC |1.0.1.1|125|266016^MDC_DIM_MMHG^MCD IIIIIFIIIIIIII20111215114505.824+0100

OBX | | MM | 150022^MDC_PRESS_BLD_NONINV_DIA^MDC |1.0.1.2|85|266016^MDC_DIM_MMHG^MCD | ||||F||||||||20111215114505.824+0100

Fig. 3. HL7v2 ORU R01 message structure including

in healthcare combined with a RESTful architecture. A FHIRResource can be described as a logical unit of information used to store or exchange data in integrated healthcare solutions. In total [15] defines about 60 resources each of them associated with one of the categories clinical, administrative or infrastructure. E.g. a patient resource is an administrative resource, whilst a medication is a clinical resource. As the specification further depicts, a resource typically consist of 3 distinctive parts. Besides the data content, a resource can also contain a narrative part, that is a representation of the resources' data in a human readable format. Additionally every resource can be extended through the use of a local extension. Figure 2 shows these fundamental parts by means of a patient resource example.

\section{A. Mapping from HL7v2 to HL7-FHIR}

CHA requires a certain HL7v2 message to be embedded in a SOAP-Request. The semantics of this message are defined by IHE as part of the Device Enterprise Communication (DEC) profile. The DEC profile [7] describes the Patient Care Device (PCD)-01 transaction as capable of transferring a measurement from a DECReporter to a DECConsumer using HL7v2 in combination with web service technologies (figure 8). Part of the research work was to identify available FHIR-Resources that are capable of resembling this HL7v2 message. The HL7 message used in connection with the PCD-01 transaction consists of a patient identification, measured data (e.g. weight, blood pressure) and the unit of measure encoded in a HL7 $\mathrm{ORU}^{\wedge} \mathrm{R} 01$ message. An example of such a HL7v2 message consisting of a measured weight value is shown in figure 3 .

Given these requirements a literature research was carried out, mainly focussing on specifications of HL7 as well as profile adaptions related to IHE. As a result [18] lists several possible candidates capable to resemble the existing PCD-01 transaction with FHIR-Resources. [18] divides these resources in 2 groups, one being Device Related Resources, the other being Non-Device Specific Resources. Furthermore the FHIR specification available at [15], defines possible mappings from previous HL7 versions and technologies. As a result based on the requirements for the contents of the message as well as possible mappings from HL7v2 messages to FHIR-Resources, the Observation and DeviceObservationReport resources were chosen as a supplement for the existing PCD-01 transaction.

1) Observation Resource: Referring to [16] an Observation contains measurements and simple assertions made about a patient, devices and other subjects (see figure 4). In order to send multiple observations at once, the FHIR specification offers the bundle-concept, which allows grouping several observations together. This bundle-concept has to be used in 


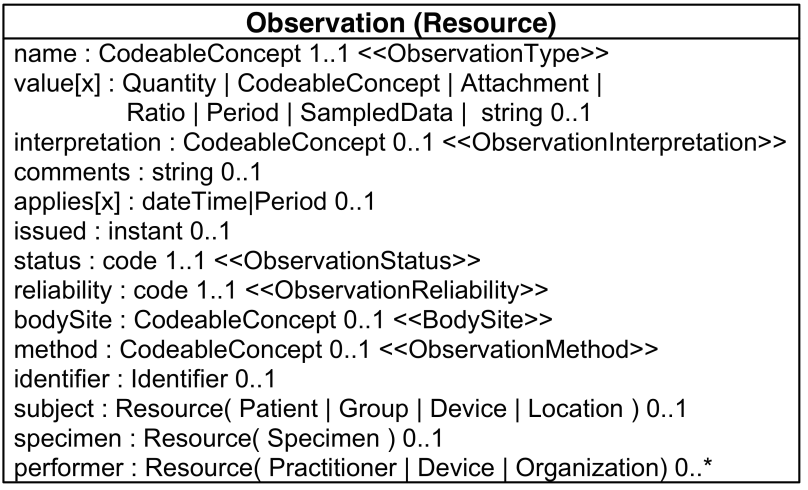

Fig. 4. Data model of Observation resource.

connection with the transactional storing of data, as defined by [16]. For example a blood pressure measurement consisting of three standalone observations, the systolic, diastolic and pulse observation, has to be bundled, whereas in case of a simple weight observation bundling can be omitted.

2) DeviceObservationReport Resource: A DeviceObservationReport describes the data produced by a device at a certain point in time. Each DeviceObservationReport contains the measurement date, a reference to a patient, a device as well as list of observations supplied from the device [16]. According to the FHIR specification a typical use case where a DeviceObservationReport should be utilized is the transfer of a vital sign from a recording device, such as a glucose meter or a blood pressure device. Figure 5 shows the data model and available references to other FHIR resources.

\section{B. Architecture}

The architecture of the telemonitoring solution introduced in [5], [6] was extended to add support for transferring both of the FHIR-Resources described above, along with the existing HL7v2 via SOAP implementation. Considering the resulting telemonitoring solution, the main focus was to conform to the proposed architecture defined by the CHA-guidelines (see figure 1), even though CHA does currently not support the exchange of FHIR-Resources. The different components of the resulting telemonitoring solution in terms of the CHAguidelines are describe in detail as follows.

1) Personal Device: A Personal Device (PD) as described by [19] is any device capable of measuring data through sensors and transfer that data according to the CHA-guidelines through the PAN-Interface. Typical examples for PDs include blood pressure meters, weight scales and glucose meters. For

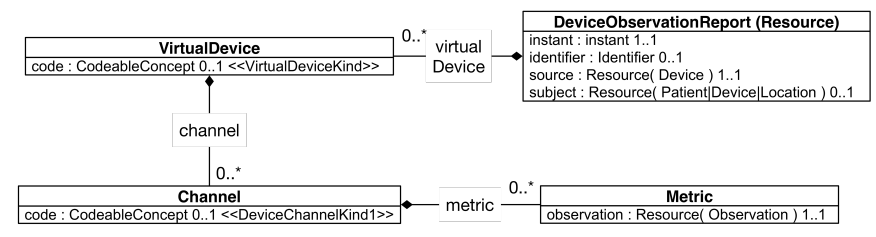

Fig. 5. Data model of DeviceObservationReport resource an its realtionship with other resources. the transport of measured data CHA supports different protocols, among those Bluetooth, which the solution presented in this paper supports. The solution covers blood pressure meters, weight scales, activity monitors and heart rate monitors conforming to the respective Bluetooth Health Device (BHD) profile.

2) Aggregation Manager: All data that is measured and transferred from a PD, is received by an Aggregation Manager (AM). The AM verifies and validates the incoming data [20]. As depicted in figure 8 the present AM consists of 3 distinctive components:

- MessageReceiver: The MessageReceiver is responsible for establishing, maintaing and releasing connections to associated personal devices. Furthermore it receives any data sent from those devices.

- MessageTransformer: The MessageTransformer converts received data into a desired target format. For example the content of measured data is transformed into a HL7v2 Message structure.

- MessageSender: After the MessageTransformer converted the received raw data, a MessageSender is used in order to encode and transfer the data over the WANInterface (see figure 1).

The AM allows for an arbitrary combination of the 3 components, e.g. there can be different MessageReceivers, each one addressed to receive data from a different PD. This design decision enables to be independent from different device types, data formats as well as service interfaces. For example the solution allows to operate different MessageSenders in parallel in order to facilitate the different types of service interfaces required. In the presented solution, the AM is implemented and deployed as an Android application.

3) Telehealth Service Center: The Telehealth Service Center (THCS) offers the required service endpoints for communicating with the AM. As specified in the CHA guidelines [19] the THCS transforms incoming HL7v2 messages into a HL7v3 CDA Personal Health Monitoring Report (PHMR). Using the IHE Cross Enterprise Document Sharing (XDS) profile the resulting report is sent over the HRN-Interface for persistent storage (see figure 1). Besides the mapping from HL7v2 to a PHMR introduced in [5], [6], an additional mapping from the HL7-FHIR resources Observation and DeviceObservationReport was added. Therefore incoming FHIR-Resources are mapped from their JSON representation to an object model defined by [15] which is further used to populate the PHMR entry-elements (see figure 6).

4) Electronic Health Record: In [21] an Electronic Health Record (EHR) is described as a program that is accessible through the internet and stores personal medical information of patients. In order to ensure interoperability and to enable access, the administration as well as the persistent storage of such medical information, IHE defines the interfaces for an EHR environment. The CHA guidelines themselves rely on these definitions from IHE in order to support a wide range of different EHR systems. Thus a personal health monitoring solution conforming to the CHA guidelines has to conform to the stipulations made by IHE, in precise it has to support the requirements defined by the XDS profile [22]. As [19] does 
not define any restrictions regarding the storage of documents, any EHR system can be used in context of the personal health monitoring solution described, as long as it offers the interfaces required.

Given this architecture the general procedure when using the telemonitoring solution can be described as follows: Each measurement sent from a PD to the AM is received by a MessageReceiver. Subsequently the received data is forwarded to MessageTransformer that decodes available data into a desired target format. The result is further transferred to a MessageSender that not only encodes data according to the requirements given through CHA and HL7-FHIR, but also sends the data to the THCS. The THCS maps the received data from the specific data encoding, whether this is HL7v2 according to CHA or as introduced above a HL7-FHIR resource to a PHMR Level 3 entry element and forwards the resulting CDA document to a PHMR system for persistent storage.

\section{Service Interfaces}

As seen in figure 8 , besides the ContinuaMessageSender that offers support for the PCD-01 transaction defined by IHE, two further MessageSenders were created. One being the MessageSender responsible for transferring measured data as FHIRObservations (FHIR-OBS), the other (FHIR-DOR) responsible for the transport of FHIR-DeviceObservationReports. Referring to the FHIR specification in [15], each resource is created using the HTTP-Post method, whereas the request body contains the measurement encoded as the respective FHIR-Resource. FHIR offers two approaches for its data representation: XML and JSON. As the intention of the presented approach is to keep data traffic at a minimum, the described solution relies on the JSON-format. The resulting architecture of the AM and the THCS as well as the respective service interfaces between them is depicted in figure 8 . In the following the three service interfaces as well as their responsibilities are summarized.

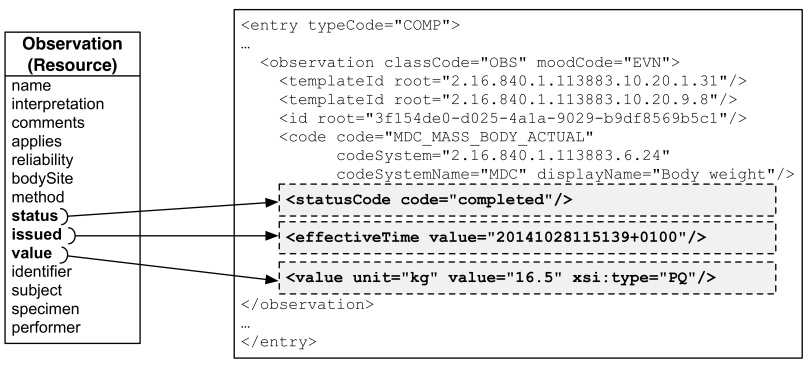

Fig. 6. Example for the mapping from a FHIR Observation resource to an entry-element in a PHMR CDA document.

resource type

http://server.org/fhir/Patient/1 endpoint identifier

Fig. 7. Example of a FHIR-Resource locator by means of a patient.

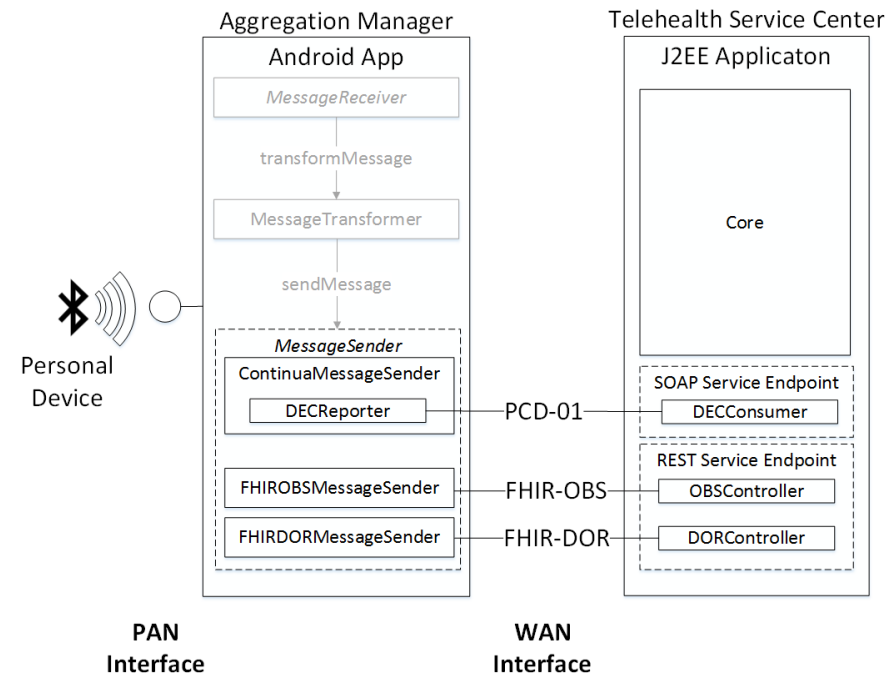

Fig. 8. Overall architecture of the extended telemonitoring solution, which offers the transfer of FHIR-Resources in addition to the PCD-01 HL7v2 via SOAP approach.

1) PCD-01: This interface implements the requirements regarding the CHA-guidelines and was introduced in [5], [6]. The effective payload of the message transferred on this interface is comprised of a HL7 ORU ^ R01 message containing measured data encoded as observation segments (see figure 3). On top of the guidelines from IHE the CHA leverages web service technologies in connection with SOAP, in order to transport such HL7v2 messages from the AM to the THCS. This requirement results mainly from the possibilities to combine different WS* specifications and technologies [19].

2) FHIR-OBS: As already described above the FHIR-OBS interface transfers measured data encoded as an Observation resource and embedded as payload in a HTTP-Request. Each Observation resource is associated with a Patient resource through the use of the FHIR-Resource reference concept [15]. Conforming to the FHIR-specification the create operation is used to add new resources which makes use of the underlying HTTP protocols post command:

POST / thcs/Observation

3) FHIR-DOR: In contrast to the FHIR-OBS interface, the FHIR-DOR interface encodes measured data in a DeviceObservationReport as proposed by [18]. Concerning the transport of the respective resource the same requirements apply as with the FHIR-OBS interface, resulting in the HTTP-Post command below:

\section{POST / thcs/DeviceobservationReport}

A full list of the supported interactions available for the FHIR-OBS and the FHIR-DOR interface is shown in table I.

\section{EvaluATION}

To evaluate this approach a test under real conditions for the duration of 5 months was conducted. In sum 68 people participated in the test, with medical conditions ranging from high blood pressure, to pulmonary hypertension and cardiac 
incidents. Each of the participants was equipped with a weight scale and an Android-based smartphone with an app preinstalled that implements the architecture of the AM described in section II-B. The app allows to receive measurements via Bluetooth from the weight scale and visualizes the received data on the display. Furthermore relating to architecture in figure 8 each received measurement is further processed as described above and sent over the available carrier network.

The goal of the evaluation was to compare the amount of data transferred for each of the three implemented service interfaces, based on real user behavior throughout the duration of the test, thus answering the question, whether the FHIR based approach helps to reduce data traffic in an environment with limited resources, compared to the HL7v2 via SOAP-based messaging implementation. To accomplish this the measurements sent from the smartphone (AM) to the server (THCS) were intercepted at the service endpoints located at the THCS. The THCS implemented as a J2EE application was deployed in an Apache Tomcat servlet container, thus offering the respective services for client devices available. For each incoming request and its subsequent response the payload size was determined. Furthermore the collected data was grouped by interface (PCD-01, FHIR-OBS, and FHIR-DOR), date and device type. As a restriction, only measurements that have been successfully processed by the system are taken into account. Hence measurements, which resulted in a fault, and/or ones that could not be processed completely due to an error, were ignored.

\section{RESULTS}

For the data collected during the evaluation of the approach, the cumulative totals for each specific interface (see figure 9) were calculated. Figure 5 shows the total data traffic recorded for weight scale devices throughout the test period. The results confirm the initial statement about the HL7v2 via SOAP

TABLE I

OVERVIEW OF THE SUPPORTED AVAILABLE INTERACTIONS ON THE SERVICE INTERFACES FHIR-OBS AND FHIR-DOR. FOR THE DEFINITION AND THE SEMANTICS OF THE INTERACTIONS REFER TO [15]

\begin{tabular}{lr|cc} 
& & FHIR-OBS & FHIR-DOR \\
\hline instance level interactions & read & $\checkmark$ & $\checkmark$ \\
vread & - & - \\
update & - & - \\
delete & - & - \\
\hline type level interactions & history & - & - \\
\hline & create & $\checkmark$ & $\checkmark$ \\
search & - & - \\
& history & - & - \\
validate & $\checkmark$ & $\checkmark$ \\
\hline system level interactions & & & - \\
& conformance & $\checkmark$ & - \\
\hline & transaction & - & - \\
& history & - & - \\
\hline
\end{tabular}

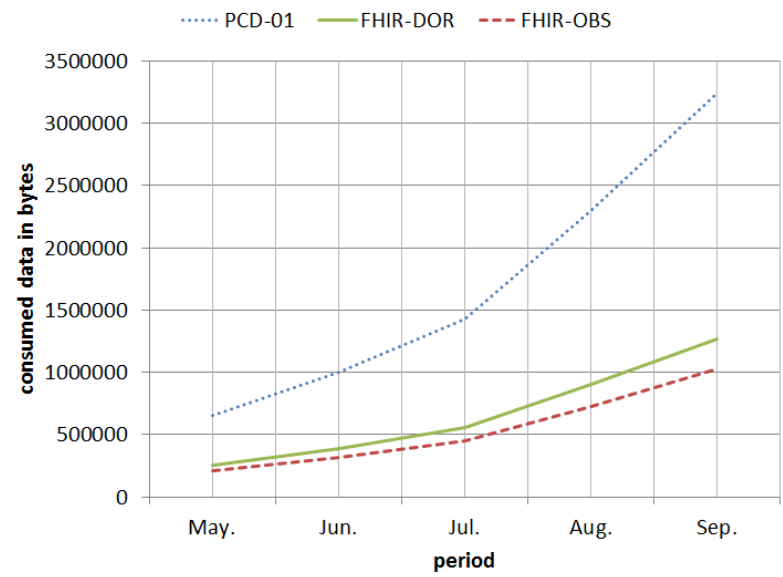

Fig. 9. Cumulative total data recorded for the test period for the device type weight scale

approach responsible for the highest data traffic. Specifically the amount of data transferred on the PCD-01 interface for the duration of 5 months is 3.2 times higher as the traffic on the FHIR-OBS interface and 2.6 times higher compared to the FHIR-DOR interface. The results for the FHIR-based interfaces (FHIR-OBS, FHIR-DOR) can be further improved by leveraging HTTPs possibility to use a compression algorithm, e.g. gzip as described in [23], leading to even lower payload sizes. This can be achieved by specifying the respective compression algorithm using Content-Enconding attribute in the HTTP header, provided that each AM and THCS support compressed HTTP processing. However the use of a compression algorithm also works on the PCD-01 interface in connection with SOAP, as long as HTTP is used as a transport protocol for the message, respectivley. Although the results look promising, it has to be said, that due to terse representation of HL7v2 messages, the estimated ratios concerning data traffic are shifting in favor of the PCD-01 interface, if several observations are sent within one request. This results from the fact that the SOAP-envelope in which the HL7v2 message is embedded does not change with the quantity of different observations in a HL7v2 message. Further investigations showed that the data traffic on the FHIR-based interfaces overhauls the PCD-01 interface when simultaniously encoded observations are involved. This difference results from the fact, that although the HL7v2 message and the respective FHIR-Resources used in the described scenario, share the same informative content, the FHIR-Resources are up to certain degree self descriptive, whereas HL7v2 messages are not.

To get insight on how the divergent user behavior affected the overall data recorded during the test period three different user categories were defined. Based on the monthly frequency of measurements available, each person was assigned to one of the defined categories. The rules for the three categories are given below with $\mathrm{x}$ being the average number of monthly weight measurements carried out by each person:

- Minimal-user $\{x \in \mathbb{N} \mid 0<x<10\}$

- Frequent-user $\{x \in \mathbb{N} \mid 10 \leq x<20\}$

- Heavy-user $\{x \in \mathbb{N} \mid x \geq 20\}$ 
Table 1 shows the resulting average amount of data contributed by each of the defined categories. Additionally using the HoltWinters approach to exponential smoothing described in [24] the estimated amount of data for the duration of one year was calculated. This was primarily done to further investigate the data usage on a carriers network and conclude possible costs for a future business case on a per year contract basis. Although the category for minimal-users consist of $82,3 \%$ of the overall users participated and is thus by far the largest category, it is only responsible for $43,9 \%$ of the overall recorded traffic, the rest being associated with the categories frequent- and heavy-user.

\section{CONCLUSION}

The telemonitoring application introduced in [5], [6] and its extension presented in this paper, offers a lightweight alternative to existing CHA compliant solutions. Given the results of the evaluation, it can be concluded that there is a significant decrease in data traffic when relying on a RESTful architecture in combination with HL7 FHIR. Furthermore due to the decreased amount of data transferred between the AM and the THSC it can be assumed that the battery life on a device with limited resources is increased, which would confirm findings of [3].

As a consequence the personal health monitoring solution described in this paper omits future support for CHAcompliant transfer of HL7v2 message via SOAP, in favor of the more flexible, lightweight and resource efficent HL7FHIR approach. This leads to the fact that CHA certification is no longer possible. However as described in [18] IHE is currently working on an extension for the DEC profile in order to support HL7-FHIR besides the traditional approach. From this it can be concluded that in near future CHA compliance can be achieved with HL7-FHIR as CHA relies on the DEC profile from IHE as already stated above. Another advantage that results from the application of the HL7-FHIR approach, is that the FHIR resource provided a level of self descriptivness, when compared to the HL7v2 approach. Hence HL7-FHIR resources follow one of the fundamental principles from [25]

TABLE II

ESTIMATED DATA CONSUMED FOR MINIMUM-, FREQUENT- AND HEAVY-USER FOR DEVICE TYPE WEIGHT SCALE. THE ESTIMATED VALUE IS CALCULATED FOR A POSSIBLE DURATION OF ONE YEAR.

\begin{tabular}{lrccr}
\hline category & & size & measurements & data (bytes) \\
\hline minimal user & & 56 & 860 & \\
& PCD-01 & & & 25894 \\
& FHIR-DOR & & 10148 \\
& FHIR-OBS & & & 8209 \\
\hline frequent user & & 8 & 612 & \\
& PCD-01 & & & 126684 \\
& FHIR-DOR & & & 49648 \\
& FHIR-OBS & & & 40163 \\
\hline heavy user & & 4 & 487 & \\
& PCD-01 & & 79016 \\
& FHIR-DOR & & 63918 \\
& FHIR-OBS & &
\end{tabular}

stating the self-descriptiveness of RESTful-architectures. The presented solution allows for flexibility when developing applications in healthcare while still maintaining interoperability. Nevertheless security related concerns have not been taken into account. Certainly, applications in healthcare and specifically a telemonitoring solution used for measuring vital signs outside a clinical setting require state-of-the art security mechanisms to ensure authenticity, authorization and authority [1], [4]. Relying on the web service profile in general and specifically on SOAP, as outlined in this paper, the CHA is able to layer any additional standards from WS-* specifications into the defined architecture [4]. In order to answer the question whether the security mechanisms offered by FHIR can compete with the security requirements defined by the $\mathrm{CHA}$, additional research has to be carried out.

\section{REFERENCES}

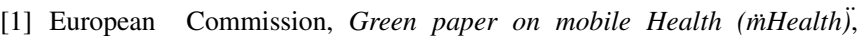
Brussels, 2014.

[2] World Health Organization, WHO, Global status report on noncommunicable diseases 2010,WHO Library Cataloguing-in-Publication Data, ISBN 978-92-4-156422-9, 2011.

[3] F. Aijaz, S.Z. Ali, M.A. Chaudhary, B. Walke, Enabling High Performance Mobile Web Services Provisioning, Vehicular Technology Conference Fall, IEEE 70th, pp.1,6, 20-23, 2009.

[4] Carroll, Randy, et al., Continua: An interoperable personal healthcare ecosystem, Pervasive Computing, IEEE 6.4. 90-94, 2007.

[5] M. Strasser, E. Helm, A. Schuler, B. Franz, H. Mayr, C. David, Telemonitoring fr mobile Pflegedienste: Entwicklung von standardkonformen Schnittstellen, eHealth2012 Health Informatics meets eHealth von der Wissenschaft zur Anwendung und zurck, Wien, sterreich, pp. 179-184, 2012

[6] M. Strasser, E. Helm, A. Schuler, M. Fuschlberger, B. Altendorfer Mobile Access to Healthcare Monitoring Data for Patients and Medical Personnel, Quality of Life through Quality of Information, Pisa, Italy, 2012.

[7] IHE International, Inc. IHE Patient Care Device (PCD) Technical Framework, Volume 1, PCD TF1, Integration Profiles, Revision 3.0 - Final Text, online resource: http://www.ihe.net/uploadedFiles/Documents/PCD/IHE_PCD_TF_Vol1.pdf, 2013, last access: 30.01.2015.

[8] Vohra Deepak, Accessing a JAX-WS web service from Android, online resource: http://www.ibm.com/developerworks/library/ws-android/ last accessed: 29.10.2014, 2011.

[9] R. Mizouni, M.A. Serhani, R. Dssouli, A. Benharref, I. Taleb, Performance Evaluation of Mobile Web Services, Web Services (ECOWS), Ninth IEEE European Conference, pp.184,191, 2011.

[10] F. Vitali, A. Amoroso, M. Rocetti, G. Marfia, RESTful Services for an Innovtive E-Health Infrastructure: A Real Case Study, 2014.

[11] G. Mulligan, D. Gracanin, A comparison of SOAP and REST implementations of a service based interaction independence middleware framework, Simulation Conference (WSC), Proceedings of the 2009 Winter, pp.1423,1432, 2009.

[12] H. Hamad, M. Saad, R. Abed, Performance Evaluation of RESTful Web Services for Mobile Devices, International Arab Journal of e-Technology, Vol. 1, No. 3, 2010

[13] P. K. Potti, S. Ahuja, K. Umapathy, Z. Prodanoff, Comparing Performance of Web Service Interaction Styles: SOAP vs. REST, Proceedings of the Conference in Information Systems Applied Research, Vol. 5, No. 2208, 2012.

[14] T. Benson, Principles of health interoperability HL7 and SNOMED, Springer London, 2012.

[15] Health Level Seven, FHIR Fast Health Interoperable Resources Specification, online resource: http://www.hl7.org/implement/standards/fhir/documentation.html, last accessed: $29.10 .2014,2011$

[16] D. Bender, K. Sartipi, HL7 FHIR: An Agile and RESTful approach to healthcare information exchange, Computer-Based Medical Systems (CBMS), IEEE 26th International Symposium, pp.326,331, 2013.

[17] Health Level Seven: HL7. Health Level Seven, http://www.hl7.org (2015), last access 30.01.2015 
[18] IHE International, Inc. IHE Patient Care Device (PCD) White Paper HL7 FHIR Device Related Resources, online resource: http://www.ihe.net/uploadedFiles/Documents/PCD/IHE_PCD_TF_Vol1.pdf, 2014, last access: 29.10.2014.

[19] Continua Health Alliance, Continua Design Guidelines, Version 2012, 2012.

[20] B. Franz, M. Buchmayr, A. Schuler, W. Kurschl, Context-enriched personal health monitoring. In Ambient Assisted Living (pp. 79-92). Springer Berlin Heidelberg, 2014.

[21] F. D. Sittig, Personal health records on the internet: a snapshot of the pioneers at the end of the 20th Century, Int J Med Inform. 2002 Apr;65(1):1-6.
[22] IHE International, Inc. IHE IT Infrastructure (ITI) Technical Framework, Volume 1, ITI TF-1, Integration Profiles, Revision 8.0 Final Text, online resource: http://www.ihe.net/Technical_Framework/upload/IHE_ITI_TF_Rev80_Vol1_FT_2011-08-19.pdf, 2011, last access: 30.01.2015.

[23] R. Fielding, J. Gettys, J. Mogul, H. Frystyk, L. Masinter, P. Leach, and T. Berners-Lee, RFC 2616: Hypertext Transfer Protocol HTTP/1.1, The Internet Society, 1999, online resource: http://www.rfceditor.org/rfc/rfc2616.txt, last access: 30.01.2015.

[24] C. Chatfield, The holt-winters forecasting procedure, Applied Statistics 264-279, 1978.

[25] R. Fielding, Architectural styles and the design of network-based software architectures. Dissertation, University of California, Irvine, 2000. 